\title{
Mathematical model for steady state current at PPO-modified micro-cylinder biosensors
}

\author{
Kodhandapani Venugopal ${ }^{1}$, Alagu Eswari ${ }^{2}$, Lakshmanan Rajendran ${ }^{2 *}$ \\ ${ }^{1}$ Department of Mathematics, J.J College of Engineering and Technology, Tiruchirappali, India; \\ ${ }^{2}$ Department of Mathematics, The Madura College, Madurai, India. \\ Email: ${ }^{*}$ raj_sms@rediffmail.com
}

Received 1 July 2011; revised 18 July 2011; accepted 3 August 2011.

\begin{abstract}
A Mathemataical model for a modified microcylinder electrode in which polyphenol oxidase ( PPO) occurs for all values of the concentration of catechol and o-quinone is analysed. This model is based on system of reaction-diffusion equations containing a non-linear term related to Michaelis Menten kinetics of the enzymatic reaction. Here a new analytical technique Homotopy Perturbation Method is used to solve the system of non-linear differential equations. that describe the diffusion coupled with a MichaelisMenten kinetics law. Here we report an analytical expressions pretaining to the concentration of catechol and $o$-quinone and corresponding current in terms of dimensionless reaction-diffusion parameters in closed form. An excellent agreement with available limiting case is noticed.
\end{abstract}

Keywords: Non-Linear Reaction/Diffusion Equation; Biosensors; Polymer-Modified Micro-Cylinder Electrode; Polyphenol Oxidase; Homotopy Perturbation Method

\section{INTRODUCTION}

Microelectrodes are increasingly being used in biosensors [1-3]. This is due to factors such as fast response times, high signal: noise ratios and the ability to operate in low conductivity media, sub-micro volume and in vivo [4]. The most commonly used microelectrode in bio-sensor is microcylinder such as carbon fibres. This is because they are cheap, readily available, their form is suited to implantation [5] and because much is known about their surface characteristics [6].

Immobilization of enzymes is used in biosensors to detect the concentration of a specific analyte as a result of the biological recognition between the analyte and the immobilized enzyme. Enzymes have been immobilized at carbon fibres by many methods. Among all the methods, layer-by-layer (LbL) self assembly process is a simple technique which may be applied to a wide range of enzymes and that it is one of the few immobilization procedures which allows control over the amount and spatial distribution of the enzyme [7]. This property is important both for constructing and modeling studies of biosensors. The layer-by-layer process was first introduced by Decher and Hong [7]. This method has been applied to planar electrodes of $\mathrm{Au}[8,9]$, carbon electrodes [10] and polystyrene latex [11-15].

To analyse the performance of biosensors of any kind, it would be useful to have a mathematical model of the electrode response. Theoretical models of enzyme electrodes give information about the mechanism and kinetics operating in the biosensor. Unlike experimental investigations of biosensors, where changing one parameter inevitably alters others, the influence of individual variables can be assessed in an idealized way. Thus, the information gained from modeling can be useful in sensor design, optimization and prediction of the electrodes response.

Recently Rijiravanich et al. [16] obtained the steady state concentration profile of $o$-quinone and dimensionless sensor response $j$ for the limiting cases of low substrate concentrations. To the best of our knowledge, no rigorous analytical solutions for the steady state concentrations for micro-cylinder biosensors for all values of the parameters have been published. In this communication, we have derived the new and simple analytical solutions of the concentration and the current for all values of parameters using the Homotopy Perturbation Method

\section{MATHEMATICAL FORMULATION OF THE PROBLEM AND ANALYSIS}

The system presented here is a cylindrical electrode which is uniformly coated by an enzyme immobilized in non-conducting material which is porous to substrate. 
The electrode is used in a stirred solution containing an excess of supporting electrolyte. The enzyme and electrode reaction are [16]:

$$
\begin{aligned}
& \mathrm{O}_{2}+2 \text { catechol } \rightarrow 2 o-\text { quinone }+2 \mathrm{H}_{2} \mathrm{O} \\
& o-\text { quinone }+2 \mathrm{H}^{+}+2 \mathrm{e}^{-} \rightarrow \text { catechol }
\end{aligned}
$$

Hence the catechol/quinone conversion forms an am- plification cycle within the enzyme film. While it is possible in principle to solve for either phenol or catechol as substrate, solving for catechol is simpler, since it involves only one enzymic conversion. The actual mechanism of that conversion is complex, and involves three different states, oxy, met, deoxy [17] i.e. (where $\mathrm{Ca}$ is catechol, $Q$ is quinone).

$$
\begin{gathered}
{[\mathrm{Cu}(\mathrm{I})-\mathrm{Cu}(\mathrm{I})]_{\text {deoxy }}+\mathrm{O}_{2}+2 \mathrm{H}_{2} \mathrm{O} \stackrel{k_{1}}{\longrightarrow}\left[\mathrm{H}_{2} \mathrm{O}-\mathrm{Cu}(\mathrm{II})-\mathrm{O}-\mathrm{O}-\mathrm{Cu}(\mathrm{II})-\mathrm{H}_{2} \mathrm{O}\right]_{\text {oxy }}} \\
{\left[\mathrm{H}_{2} \mathrm{O}-\mathrm{Cu}(\mathrm{II})-\mathrm{O}-\mathrm{O}-\mathrm{Cu}(\mathrm{II})-\mathrm{H}_{2} \mathrm{O}\right]_{\text {oxy }} \stackrel{k_{2}}{\longrightarrow}[\mathrm{Cu}(\mathrm{II})-\mathrm{Ca}-\mathrm{Cu}(\mathrm{II})]_{\text {met }}+2 \mathrm{H}_{2} \mathrm{O}+2 \mathrm{H}^{+}} \\
{[\mathrm{Cu}(\mathrm{II})-\mathrm{Ca}-\mathrm{Cu}(\mathrm{II})]_{\text {met }} \stackrel{k_{3}}{\longrightarrow}[\mathrm{Cu}(\mathrm{I})-\mathrm{Cu}(\mathrm{I})]_{\text {deoxy }}+Q}
\end{gathered}
$$

It is assumed that the enzyme concentration is uniform and that the enzyme reaction follows Michaelis-Menten kinetics, in which case the reaction in the film is [18]

$$
S+E_{1} \underset{k_{2}}{\stackrel{k_{1}}{\Leftrightarrow}}\left[E_{1} S\right] \stackrel{k_{c a t}}{\longrightarrow} P+E_{2}
$$

where

$$
k_{c a t}=k_{1} c_{O_{2}} \text { and } K_{M}=\frac{k_{1}\left(k_{2}+k_{3}\right) c_{O_{2}}}{k_{2} k_{3}}
$$

are the rate constant and Michaelis-Menten constant. The model of a cylindrical electrode modified with both an enzyme and conducting sites/particles (circles) is shown in Figure 1. The mass balance for catechol $c_{C}$ can be written in cylindrical coordinates as follows:

$$
\frac{D_{C}}{r} \frac{\mathrm{d}}{\mathrm{d} r}\left(r \frac{\mathrm{d} c_{C}}{\mathrm{~d} r}\right)-\frac{k_{c a t} c_{E} c_{C}}{c_{C}+K_{M}}=0
$$

where $c_{C}$ is the concentration profile of catechol, $c_{E}$ is the concentration profile of enzyme, $D_{C}$ and $D_{Q}$ are its diffusion coefficients, and $K_{M}$ is the Michaelis constant and $c_{Q}$ is the concentration profile of quinone. Then the equation of continuum for quinone is generally expressed in the steady-state by [16]

$$
\frac{D_{Q}}{r} \frac{\mathrm{d}}{\mathrm{d} r}\left(r \frac{\mathrm{d} c_{Q}}{\mathrm{~d} r}\right)+\frac{k_{c a t} c_{E} c_{C}}{c_{C}+K_{M}}=0
$$

At the electrode surface $\left(r_{0}\right)$ and at the film surface $\left(r_{1}\right)$ the boundary conditions are given by [16]

$$
\begin{array}{lll}
r=r_{0}: & c_{C}=c_{C}^{*}, & c_{Q}=0 \\
r=r_{1}: & c_{C}=c_{C}^{*}, & c_{Q}=0
\end{array}
$$

where $c_{C}^{*}$ is the bulk concentration of catechol scaled by the partition coefficient of the enzyme film. Adding the Eqs.8 and $\mathbf{9}$ and integrating with boundary condition (10), yields

$$
\frac{c_{C}(r)}{c_{C}^{*}}+\frac{D_{Q} c_{Q}(r)}{D_{C} c_{C}^{*}}=1
$$

The steady-state current can be given as [16]:

$$
\frac{I}{n F}=2 \pi L r_{0} D_{Q}\left(\mathrm{~d} c_{Q} / \mathrm{d} r\right)_{r=r_{0}}
$$

We introduce the following set of dimensionless variables:

$$
\begin{gathered}
C=\frac{c_{C}}{c_{C}^{*}}, Q=\frac{c_{Q}}{c_{C}^{*}}, \quad R=\frac{r}{r_{0}}, \alpha=\frac{c_{C}^{*}}{K_{M}}, \gamma_{E}=\frac{k_{c a t} c_{E} r_{0}^{2}}{D_{C} K_{M}}, \\
\gamma_{S}=\frac{k_{c a t} c_{E} r_{0}^{2}}{D_{Q} K_{M}}, \frac{D_{Q}}{D_{C}}=\frac{\gamma_{E}}{\gamma_{S}}
\end{gathered}
$$

where $C$ and $Q$ are the dimensionless concentration of the catechol and $o$-quinone. $R$ is the dimensionless distance parameter. $\gamma_{E}, \gamma_{S}$ and $\alpha$ are the dimensionless reaction-diffusion parameters and saturation parameter [16].

$$
\begin{aligned}
& \frac{\mathrm{d}^{2} C}{\mathrm{~d} R^{2}}+\frac{1}{R} \frac{\mathrm{d} C}{\mathrm{~d} R}-\frac{\gamma_{E} C}{1+\alpha C}=0 \\
& \frac{\mathrm{d}^{2} Q}{\mathrm{~d} R^{2}}+\frac{1}{R} \frac{\mathrm{d} Q}{\mathrm{~d} R}+\frac{\gamma_{S} C}{1+\alpha C}=0
\end{aligned}
$$

The boundary conditions are represented as follows:

$$
\begin{array}{cl}
C=1, Q=0 & \text { when } R=1 \\
C=1, Q=0 & \text { when } R=r_{1} / r_{0}
\end{array}
$$

The dimensionless current at the micro-cylinder electrode can be given as follows:

$$
\psi=I / n F L D_{Q} c_{C}^{*}=2 \pi(\mathrm{d} Q / \mathrm{d} R)_{R=1}
$$

\section{ANALYTICAL SOLUTIONS OF THE CONCENTRATIONS AND THE CURRENT USING THE HOMOTOPY PERTURBATION METHOD}

Nonlinear phenomena play a crucial role in applied 


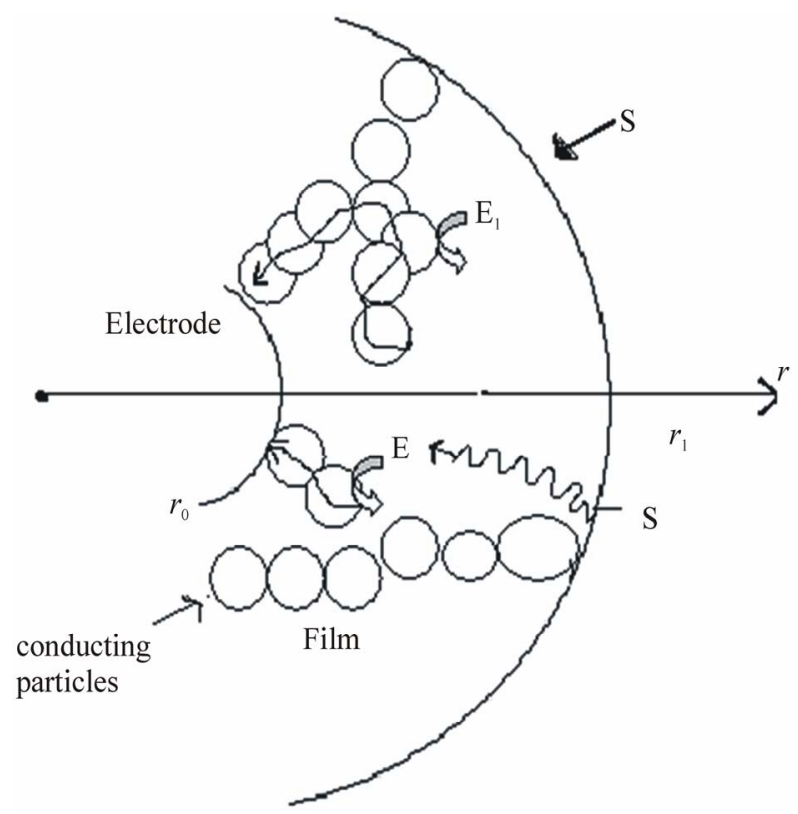

Figure 1. Illustration of the model of a cylindrical electrode modified with both an enzyme and conducting sites/particles (circles).

mathematics and chemistry. Construction of particular exact solutions for these equations remains an important problem. Finding exact solutions that have a physical, chemical or biological interpretation is of fundamental importance. This model is based on steady-state system of diffusion equations containing a non-linear reaction term related to Michaelis-Menten kinetics of the enzymatic reactions. It is not possible to solve these equations using standard analytical technique. In the past, many authors mainly had paid attention to study solution of nonlinear equations by using various methods, such as Backlund transformation [19], Darboux transformation [20], Inverse scattering method [21], Bilinear method [22], The tanh method [23], Variational iteration method [24] and Homotopy Perturbation Method [25-28] etc. The Homotopy Perturbation Method [25-28] has been extensively worked out over a number of years by numerous authors. The Homotopy Perturbation Method was first proposed by He [24-26] and was successfully applied to autonomous ordinary differential equations to nonlinear polycrystalline solids and other fields.

Recently Meena and Rajendran [29], Anitha et al. [30] and Manimozhi et al. [31] implemented Homotopy perturbation method to give approximate and analytical solutions of nonlinear reaction-diffusion equations con- taining a nonlinear term related to Michaelis-Menten kinetic of the enzymatic reaction. Eswari et al. in series $[32,33]$ solved the coupled non linear diffusion equations analytically for the microdisk and micro-cylinder enzyme electrode when a product from an immobilized enzyme reacts with the electrode. Using Homotopy Perturbation Method (see Appendix B), we can obtain the following solutions to the Eqs.14 to 15 .

$$
\begin{gathered}
C(R)=1+\left[\frac{\gamma_{E} R^{2}-\gamma_{E}\left(1+r_{1} / r_{0}\right) R+\gamma_{E}\left(r_{1} / r_{0}\right)}{2(1+\alpha)}\right] \\
Q(R)=\left[\frac{-\gamma_{S} R^{2}+\gamma_{S}\left(1+r_{1} / r_{0}\right) R-\gamma_{S}\left(r_{1} / r_{0}\right)}{2(1+\alpha)}\right]
\end{gathered}
$$

The Eqs.19-20 satisfies the boundary conditions (16) to (17). These equations represent the new and simple analytical expression of the concentration of catechol and $o$-quinone for all possible values of the parameters $\gamma_{E}, \gamma_{S}, \alpha$ and $r_{1} / r_{0}$. The Eqs.19 and 20 also satisfy the relation $C(R)+\left(\gamma_{E} / \gamma_{S}\right) Q(R)=1$. From Eqs.19 and 20, we can obtain the dimensionless current, which is as follows:

$$
\psi=I / n F L D_{Q} c_{C}^{*}=2 \pi\left[\frac{\gamma_{S}\left(1+r_{1} / r_{0}\right)-2 \gamma_{S}}{2(1+\alpha)}\right]
$$

Eq. (21) represents the new and closed form of an analytical expression for the current for all possible values of parameters.

\subsection{Limiting Cases for Unsaturated (First Order) Catalytic Kinetics}

In this case, the catechol concentration $c_{C}$ is less than Michaelis constant $K_{M}$. Now the Eqs.8 and $\mathbf{9}$ reduce to the following forms:

$$
\begin{gathered}
\frac{D_{C}}{r} \frac{\mathrm{d}}{\mathrm{d} r}\left(r \frac{\mathrm{d} c_{C}}{\mathrm{~d} r}\right)-\frac{k_{c a t} c_{E} c_{C}}{K_{M}}=0 \\
\frac{D_{Q}}{r} \frac{\mathrm{d}}{\mathrm{d} r}\left(r \frac{\mathrm{d} c_{Q}}{\mathrm{~d} r}\right)+\frac{k_{c a t} c_{E} c_{C}}{K_{M}}=0
\end{gathered}
$$

By solving the Eq.22 using the boundary condition (Eq.10), the concentration of catechol $c_{C}$ can be obtained in the form of modified Bessel functions of zeroth order $I_{0}\left(\chi_{r}\right)$ and $K_{0}(\chi r)$.

$$
c_{C}(r) / c_{C}^{*}=\left[\frac{I_{0}(\chi r)\left[K_{0}\left(\chi r_{0}\right)-K_{0}\left(\chi r_{1}\right)\right]+K_{0}(\chi r)\left[I_{0}\left(\chi r_{1}\right)-I_{0}\left(\chi r_{0}\right)\right]}{K_{0}\left(\chi r_{0}\right) I_{0}\left(\chi r_{1}\right)-K_{0}\left(\chi r_{1}\right) I_{0}\left(\chi r_{0}\right)}\right]
$$


where

$$
\begin{gathered}
\chi^{2}=k_{\text {cat }} c_{E} / D_{C} K_{M} \quad(25) \quad \text { centration } c_{Q} \\
\frac{D_{Q} c_{Q}(r)}{D_{C} c_{C}^{*}}=1-\left[\frac{I_{0}(\chi r)\left[K_{0}\left(\chi r_{0}\right)-K_{0}\left(\chi r_{1}\right)\right]+K_{0}(\chi r)\left[I_{0}\left(\chi r_{1}\right)-I_{0}\left(\chi r_{0}\right)\right]}{K_{0}\left(\chi r_{0}\right) I_{0}\left(\chi r_{1}\right)-K_{0}\left(\chi r_{1}\right) I_{0}\left(\chi r_{0}\right)}\right]
\end{gathered}
$$

Inserting Eqs.24 into Eqs.11, we can obtain the con-

The sensor response $j$ in terms of modified Bessel function of zeroth order can be obtained as follows:

$$
\begin{aligned}
& j=\frac{I}{n F L D_{C} c_{C}^{*}} \\
& =\frac{2 \pi \chi r_{0}}{\left[K_{0}\left(\chi r_{0}\right) I_{0}\left(\chi r_{1}\right)-K_{0}\left(\chi r_{1}\right) I_{0}\left(\chi r_{0}\right)\right]}\left\{K_{1}\left(\chi r_{0}\right)\left[I_{0}\left(\chi r_{1}\right)-I_{0}\left(\chi r_{0}\right)\right]-I_{1}\left(\chi r_{0}\right)\left[K_{0}\left(\chi r_{0}\right)-K_{0}\left(\chi r_{1}\right)\right]\right\}
\end{aligned}
$$

\section{COMPARISON WITH LIMITING CASE WORK OF RIJIRAVANICH ET AL. [16]}

Recently, they [16] have derived the analytical expres- sion of the steady- state concentration $c_{Q}$ (Eq.28 and sensor response $j$ (Eqs.28 and 29) in integral form for the limiting case $c_{C}<K_{M}$.

$$
\begin{aligned}
& \frac{D_{Q} c_{Q}(r)}{D_{C} c_{C}^{*}}=g \chi\left\{-f \int_{r_{0}}^{r} I_{1}(\chi r) \mathrm{d} r+\int_{r_{0}}^{r} K_{1}(\chi r) \mathrm{d} r+\frac{\ln \left(r / r_{0}\right)}{\ln \left(r_{1} / r_{0}\right)}\left[f \int_{r_{0}}^{n_{1}} I_{1}(\chi r) \mathrm{d} r-\int_{r_{0}}^{n_{1}} K_{1}(\chi r) \mathrm{d} r\right]\right\} \\
& j=\frac{I}{n F L D_{C} c_{C}^{*}}=2 \pi \chi g \times\left\{r_{0}\left[-f I_{1}\left(\chi r_{0}\right)+K_{1}\left(\chi r_{0}\right)\right]+\frac{1}{\ln \left(r_{1} / r_{0}\right)}\left[f \int_{r_{0}}^{r_{1}} I_{1}(\chi r) \mathrm{d} r-\int_{r_{0}}^{r_{1}} K_{1}(\chi r) \mathrm{d} r\right]\right\}
\end{aligned}
$$

where

$$
g=1 /\left[f I_{0}\left(\chi r_{0}\right)+K_{0}\left(\chi r_{0}\right)\right], \quad f=\left[K_{0}\left(\chi r_{0}\right)-K_{0}\left(\chi r_{1}\right)\right] /\left[I_{0}\left(\chi r_{1}\right)-I_{0}\left(\chi r_{0}\right)\right] .
$$

Rijiravanich et al. [16] obtained the empirical expression of the current

$$
j=2 \pi x^{q} \tanh \left[(x / 2)\left(\alpha_{1}-1\right)\right]^{p}
$$

where $p$ and $q$ are empirical constants and $\alpha_{1}=r_{1} / r_{0}$. The value of $p$ and $q$ are given for various values of $x\left(=\chi r_{0}\right)$ in the Tables 1-3. This empirical expression is compared our simple closed analytical expression Eq.27, in Tables 2-3. The average relative difference between our Eq.27 and the empirical expression Eq.30 is $0.71 \%$ when $\alpha_{1}=1.5$ and $0.59 \%$ when $\alpha_{1}=5$.

\section{DISCUSSION}

Figures 2 and 3 shows the dimensionless concentration profile of catechol $C(R)$ using Eq.19 for all

Table 1. Values of $p$ and $q$ which fit Eq.30 to Eq.29 with $<5 \%$ error [16].

\begin{tabular}{ccc}
\hline$x$ & $p$ & $q$ \\
\hline $9.0-7.0$ & 1.00 & 1.01 \\
$6.0-4.0$ & 1.03 & 1.05 \\
3.0 & 1.04 & 1.10 \\
2.0 & 1.02 & $1.14^{a} / 1.25^{b}$ \\
\hline
\end{tabular}

a Valid for $\alpha_{1} \leq 2.0$; b Valid for $\alpha_{1}>2.0$ various values of the parameters $\gamma_{S}, \gamma_{E}, r_{1} / r_{0}$ and $\alpha$. Thus it is concluded that there is a simultaneous increase in the values of the concentration of catechol as well as in saturated parameter $\alpha$ for small values of $\gamma_{E}$. Also the value of catechol concentration $C$ is approximately equal to 1 when $R=1$ and $R=r_{1} / r_{0}$ for all values of $\alpha$ and $\gamma_{\mathrm{E}}$.

Figures 4 and 5 show the concentration profile of $o$-quinone $Q(R)$ in $R$ space for various values of $\alpha$ and $\gamma_{S}$ calculated using Eq.20. The plot was constructed for $r_{1} / r_{0}=1.5$ and 5 . From these figures, it is confirmed that the value of the concentration of $o$ quinone increases when $\gamma_{S} \geq 0.1$ for small values of $\alpha$. From the Figures 2-5, we can observed that the dimensionless concentration of catechol should vary between 0 and 1 . Because catechol is converted to $o-$ quinone, the $o$ - quinone concentration should be the inverse of catechol. The substrate catechol $C$ is minimum and product $o$-quinone $Q$ is maximum when $R=\left(0.5+r_{1} / 2 r_{0}\right)$ for all values of $\gamma_{S}$ and $\alpha$. The minimum value of concentration profile of catechol is

$$
C_{\min }=\frac{8+8 \alpha-\gamma_{E}+2 \gamma_{E} \alpha_{1}-\gamma_{E} \alpha_{1}^{2}}{8(1+\alpha)}
$$


Table 2. Comparison of dimensionless sensor response $j$ for various values of $\chi r_{0}$ using Eqs.27 and $\mathbf{3 0}$ when thickness of the film $\left(\alpha_{1}=r_{1} / r_{0}=5\right)$.

\begin{tabular}{ccccccc}
\hline$x\left(=\chi r_{0}\right)$ & $\alpha_{1}=r_{1} / r_{0}$ & $p$ & $q$ & Eq. (30) [16] & Eq. (27) This work & Error \% \\
\hline 9 & 5 & 1 & 1.01 & 57.78 & 57.78 & 0.00 \\
8 & 5 & 1 & 1.01 & 51.30 & 51.30 & 0.00 \\
7 & 5 & 1 & 1.01 & 44.82 & 44.78 & 0.09 \\
5 & 5 & 1.03 & 1.05 & 34.03 & 34.01 & 0.06 \\
4 & 5 & 1.03 & 1.05 & 26.92 & 25.95 & 3.77 \\
3 & 5 & 1.04 & 1.10 & 21.03 & 14.99 & 0.19 \\
2 & 5 & 1.02 & 1.25 & 14.93 & & 0.01 \\
& & & & & 0.59 \\
\hline
\end{tabular}

Table 3. Comparison of dimensionless sensor response $j$ for various values of $\chi r_{0}$ using Eqs.27 and $\mathbf{3 0}$ when thickness of the film $\left(\alpha_{1}=r_{1} / r_{0}=1.5\right)$.

\begin{tabular}{ccccccc}
\hline$x\left(=\chi r_{0}\right)$ & $\alpha_{1}=r_{1} / r_{0}$ & $p$ & $q$ & Eq. $(30)[16]$ & Eq. (27) This work & Error \% \\
\hline 9 & 1.5 & 1 & 1.01 & 56.51 & 56.51 & 0.00 \\
8 & 1.5 & 1 & 1.01 & 49.45 & 49.45 & 0.01 \\
7 & 1.5 & 1 & 1.01 & 42.20 & 42.19 & 0.02 \\
5 & 1.5 & 1.03 & 1.05 & 28.62 & 27.60 & 3.67 \\
4 & 1.5 & 1.03 & 1.05 & 20.27 & 13.43 & 0.80 \\
3 & 1.5 & 1.04 & 1.10 & 13.09 & 6.15 & 0.45 \\
2 & 1.5 & 1.02 & 1.14 & 6.32 & & 0.01 \\
& & Average \% deviation & & & 0.71 \\
\hline
\end{tabular}

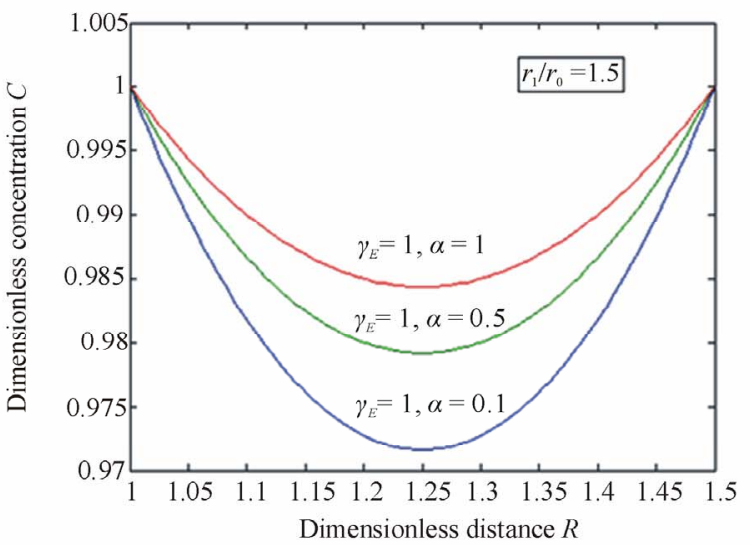

(a)

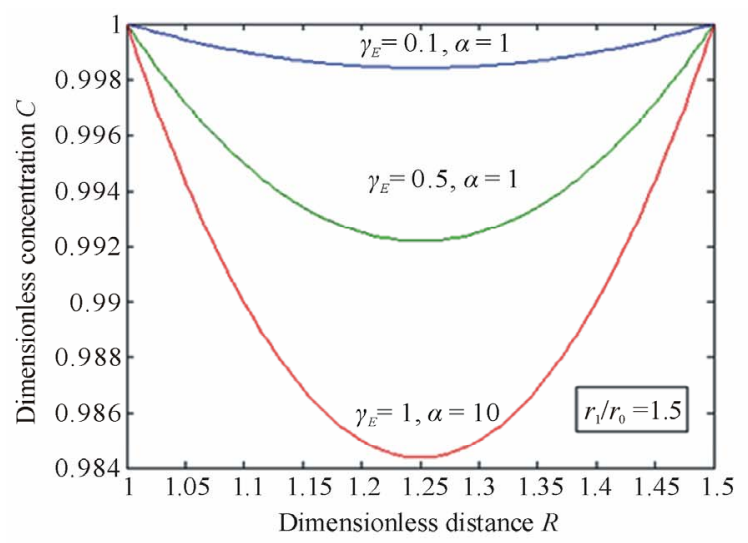

(c)

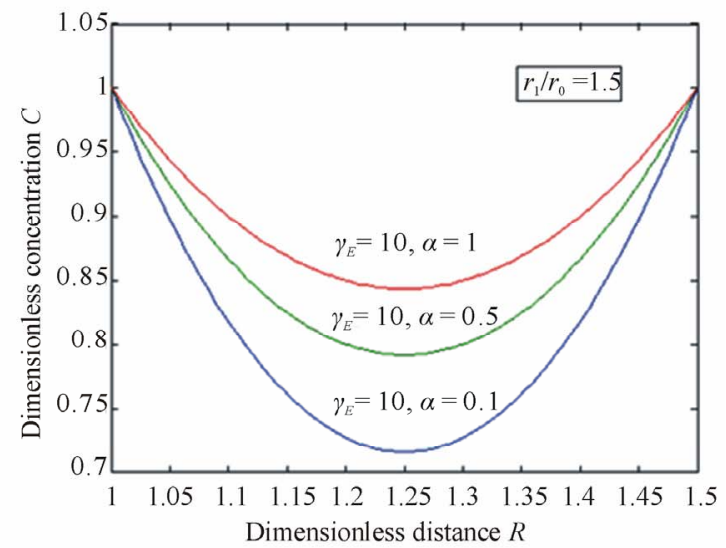

(b)

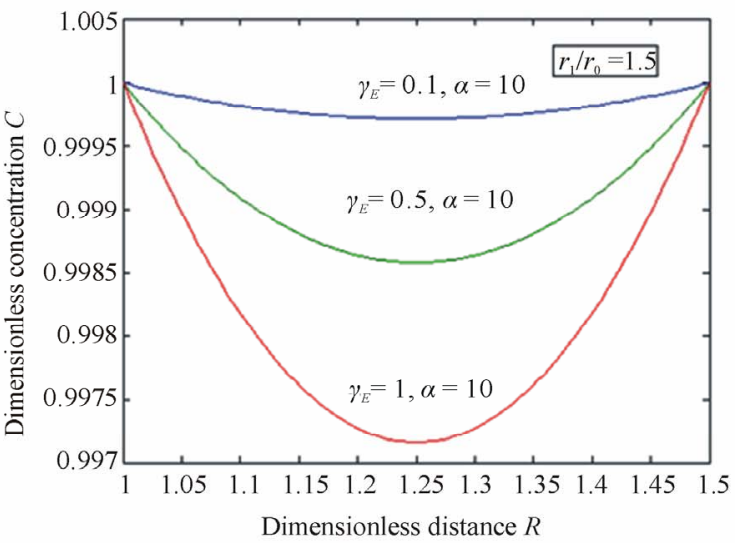

(d)

Figure 2. Typical normalized steady-state concentration profile of catechol $C(R)$ plotted from Eq.19 for different values of parameters $\gamma_{E}$ and $\alpha$ when $r_{1} / r_{0}=1.5$. 


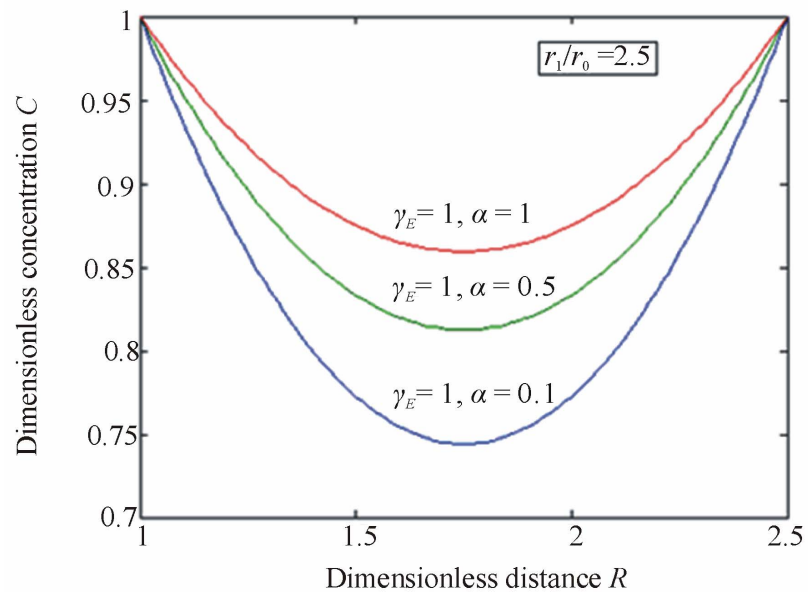

(a)

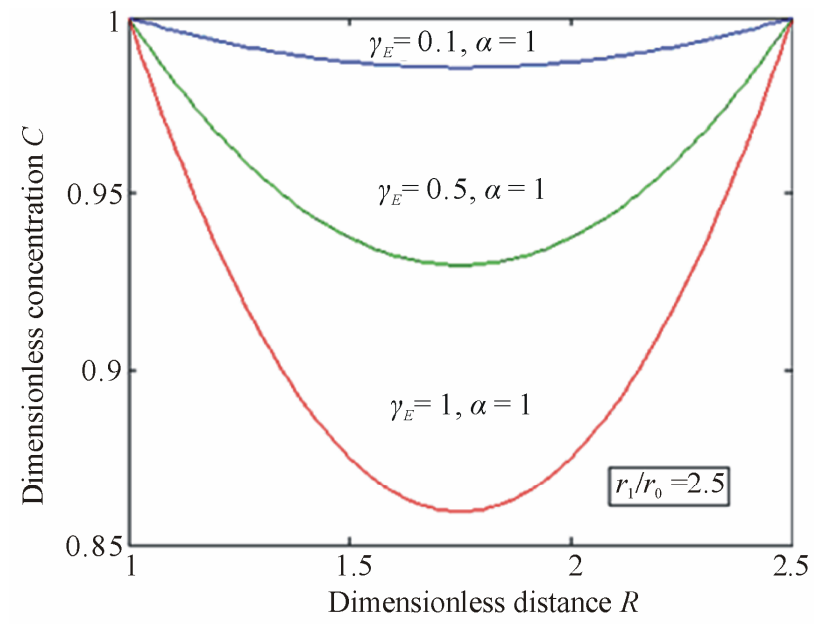

(c)

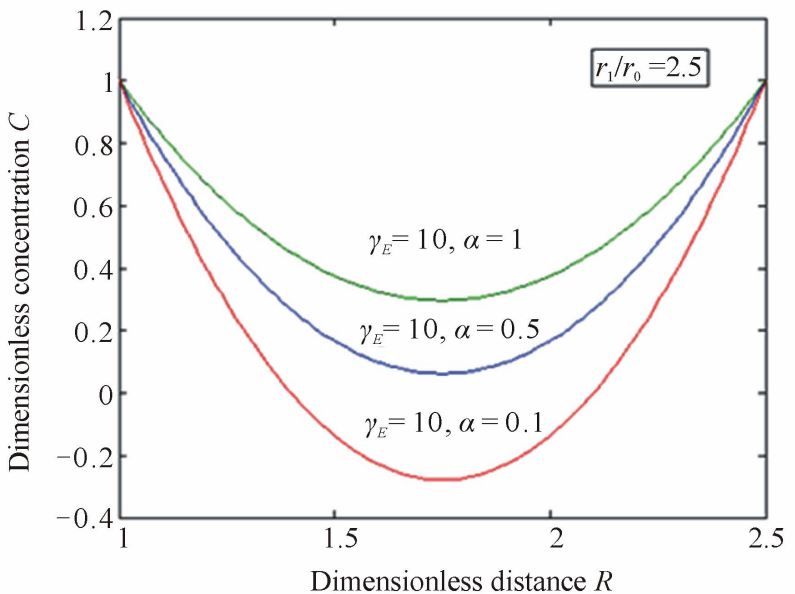

(b)

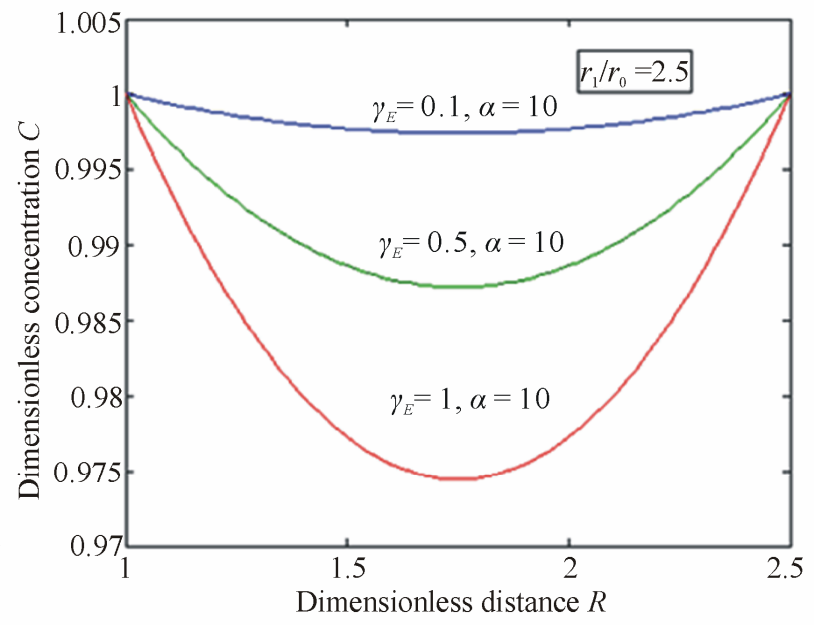

(d)

Figure 3. Typical normalized steady-state concentration profile of $C(R)$ plotted from Eq.19 for different values of parameters $\gamma_{E}$ and $\alpha$ when $r_{1} / r_{0}=2.5$.

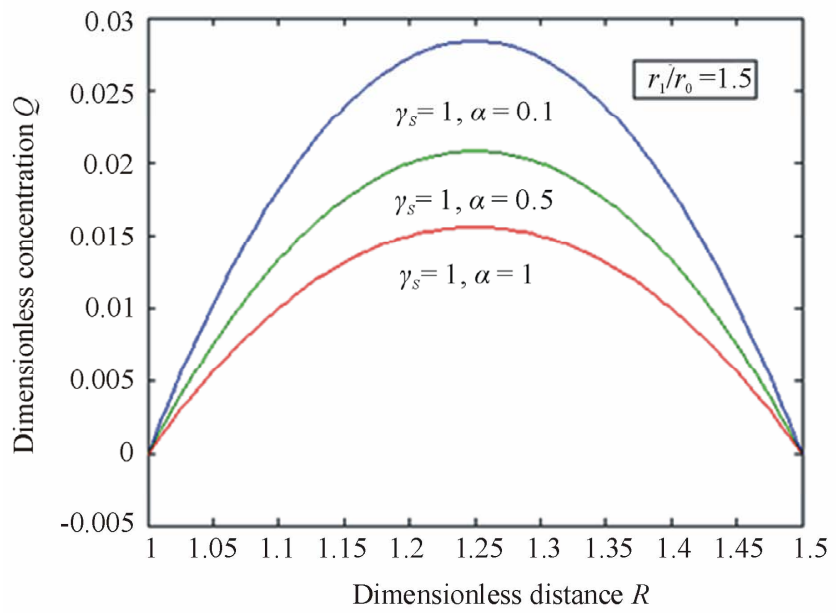

(a)

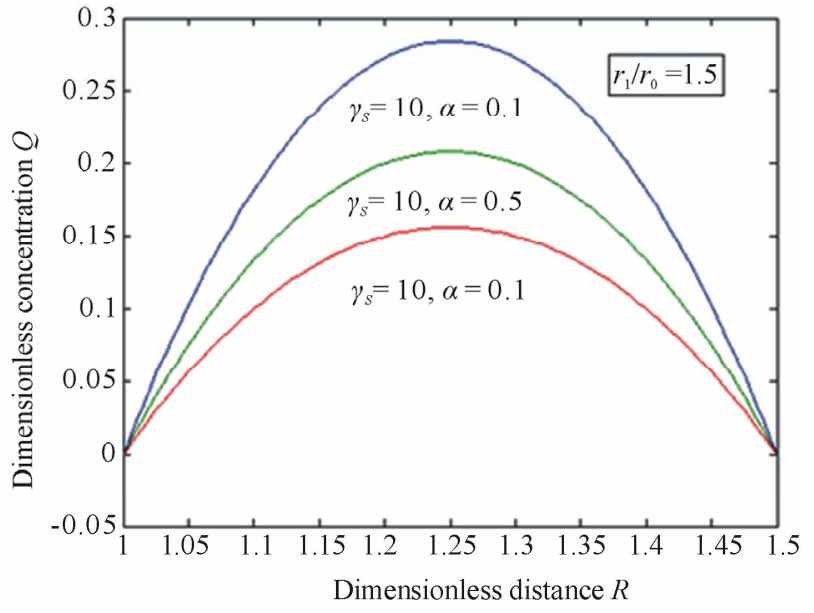

(b) 


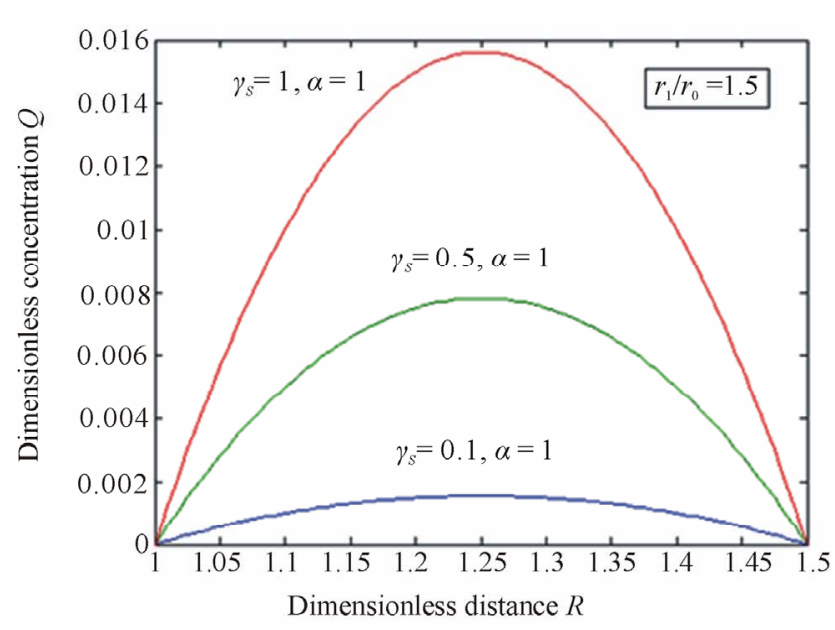

(c)

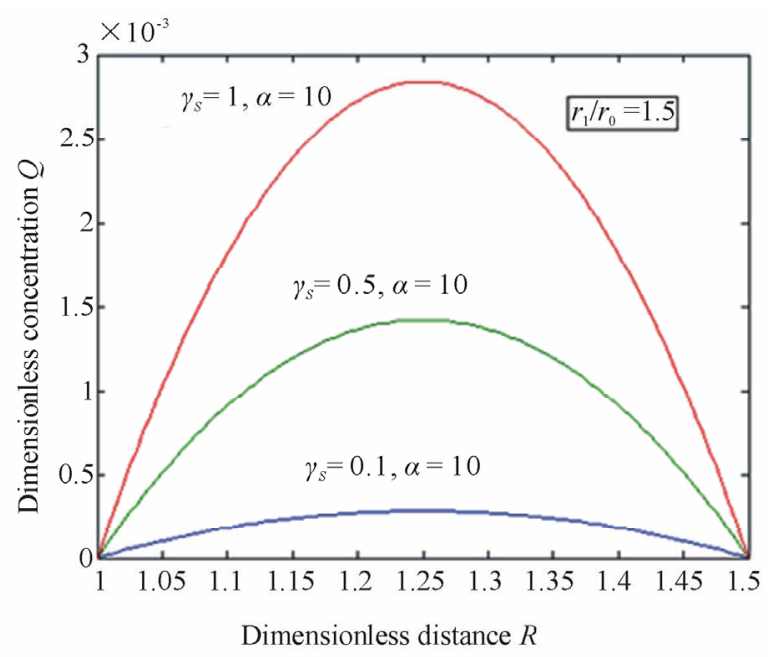

(d)

Figure 4. Typical normalized steady-state concentration profile of $Q(R)$ plotted from Eq.20 for different values of parameters $\gamma_{E}$ and $\alpha$ when $r_{1} / r_{0}=1.5$.

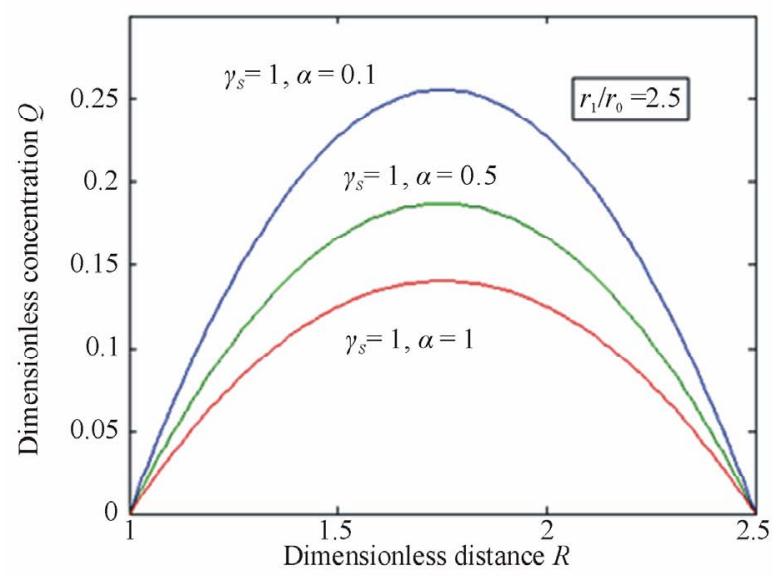

(a)

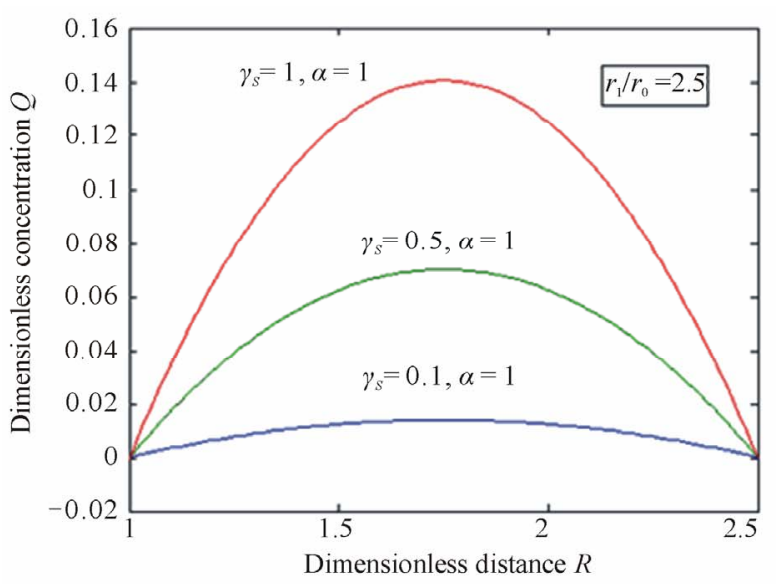

(c)

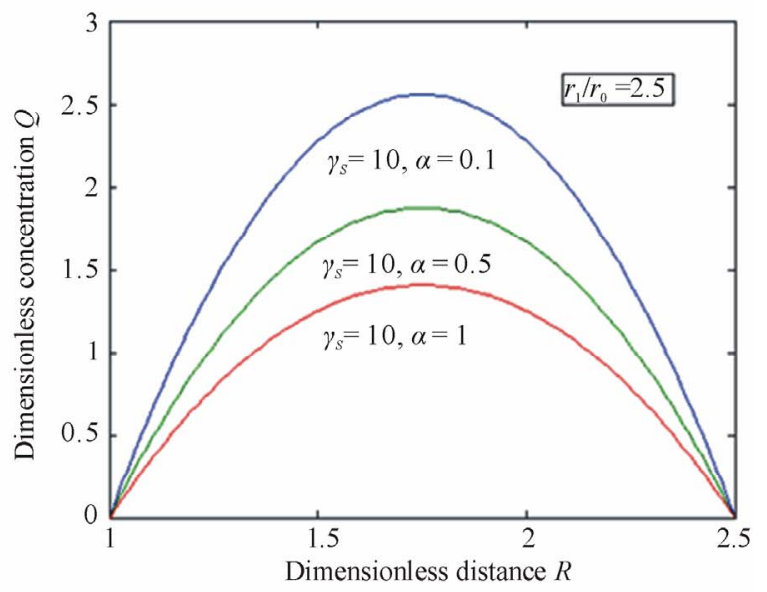

(b)

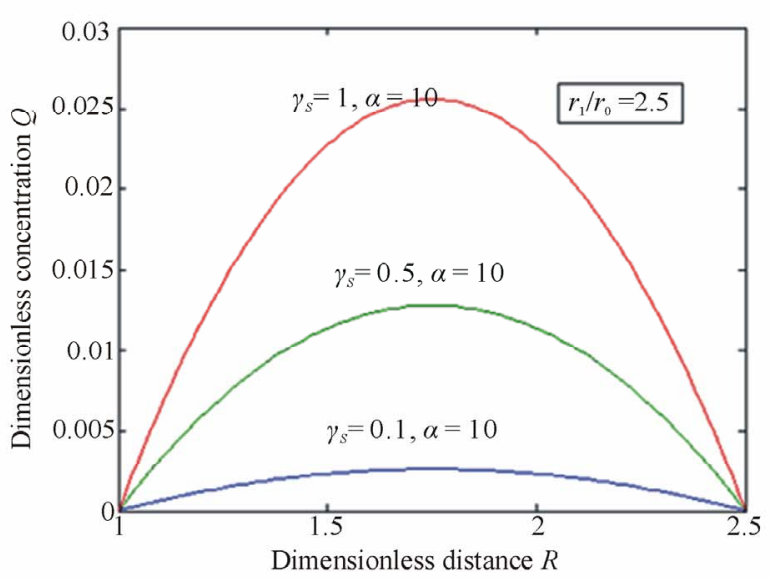

(d)

Figure 5. Typical normalized steady-state concentration profile of $Q(R)$ plotted from Eq.20 for different values of parameters $\gamma_{E}$ and $\alpha$ when $r_{1} / r_{0}=2.5$. 


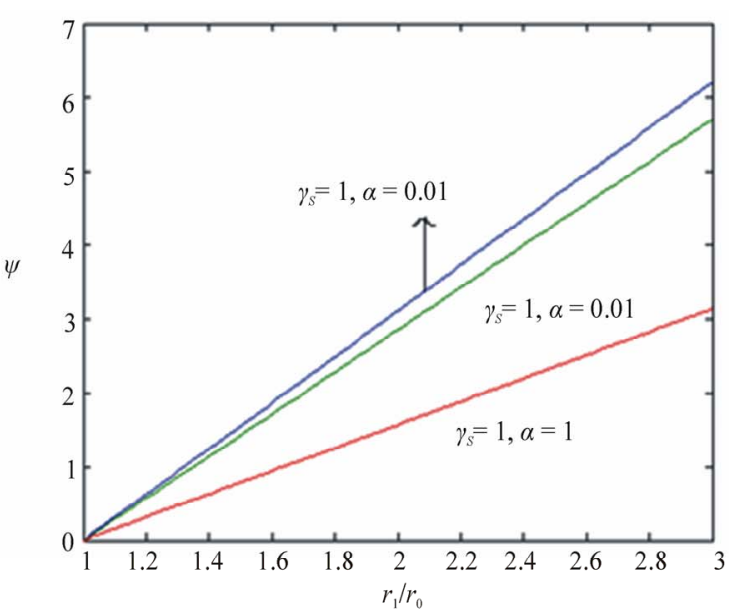

(a)

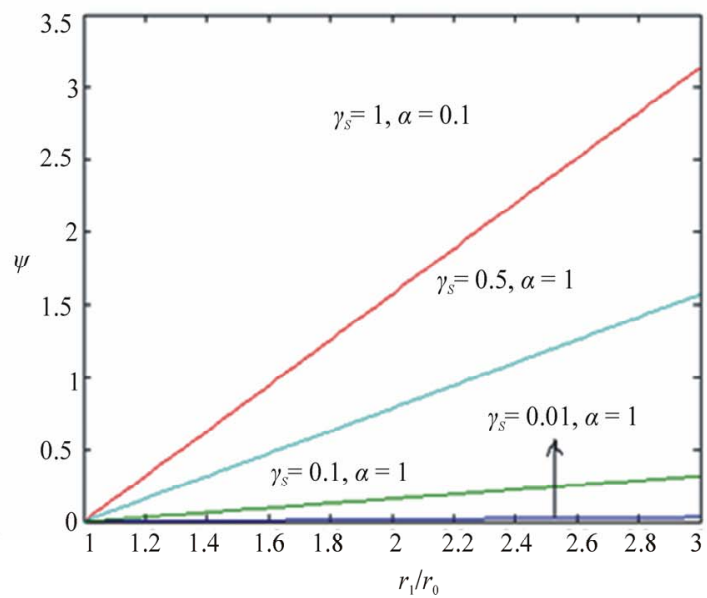

(b)

Figure 6. Plot of dimensionless current $\psi$ versus $r_{1} / r_{0}$. Current is calculated in the Eq.21.

and the maximum value of concentration profile of quinone is

$$
Q_{\max }=\frac{\gamma_{S}\left(1-2 \alpha_{1}+\alpha_{1}^{2}\right)}{8(1+\alpha)}
$$

where $r_{1} / r_{0}=\alpha_{1}$. The dimensionless current $\psi$ versus $r_{1} / r_{0}$ using Eq.21 is plotted in Figure 6. The value of current $\psi$ increases when thickness of the film $r_{1} / r_{0}$ and dimensionless reaction-diffusion parameter $\gamma_{S}$ is increases or decreases.

\section{CONCLUSIONS}

A non-linear time independent ordinary differential equation has been formulated and solved analytically. Analytical expression for the concentration of catechol and $o$-quinone and steady state current are derived by contains significant non-linear contributions using the Homotopy Perturbation Method. The primary result of this work is simple approximate calculation of concentration of catechol, $o$-quinone and current for all values of $\gamma_{E}, \gamma_{S}, \alpha$ and $r_{1} / r_{0}$ and $\chi r_{0}$. Formerly in polyphenol oxidase micro-cylinder biosensor models are [16] have only considered the first order kinetics of the enzyme and therefore could only be applied to the sensor's linear range. However, in this paper, calibration curves of many of the catechol/phenol biosensors contain most important non-linear contributions are reported. Also, the length of the linear range is an important analytical parameter. In developing a sensor, experimental scientists would like this range to cover all concentrations expected in actual samples, as this makes calibration of the sensor in the field much easier. In Tables 2-3, our analytical results are compared with limiting case of first order catalytic kinetics [16] results, which yield a good agreement with the previous limiting case results.

\section{ACKNOWLEDGEMENTS}

This work was supported by the Council of Scientific and Industrial Research (CSIR No.: 01(2442)/10/EMR-II), Government of India. The authors also thank Mr. M. S. Meenakshisundaram, Secretary, The Madura College Board, Dr. R. Murali, The Principal,nd S.Thia- garajan, Head of the Department, Madura College, Madurai, India for their constant encouragement. The authors K. Venugopal and A. Eswari are very thankful to the Manonmaniam Sundaranar University, Tirunelveli for allowing to do the research work.

\section{REFERENCES}

[1] Revzin, A.F., Sirkar, K., Simonian, A. and Pishko, M.V. (2002) Glucose, lactate and pyruvate biosensor arrays based on redox polymer/oxidoreductase nanocomposite thin films deposited on photolithographically patterned gold electrodes. Sensor and Actuators B, 81, 359 . doi:10.1016/S0925-4005(01)00982-0

[2] Shi, G., Liu, M., Zhu, M., Zhou, T., Chen, J., Jin, L. and Jin, J.-Y. (2002) The study of nafion/xanthine oxidase/au colloid chemically modified biosensor and its application in the determination of hypoxanthine in myocardial cells in vivo. Analyst, 127, 396. doi:10.1039/b108462n

[3] Gue, A.-M., Tap, H., Gros, P. and Maury, F. (2002) A miniaturized silicon based enzymatic biosensor: Towards a generic structure and technology for multi-analytes assays. Sensor and Actuators $B, \mathbf{8 2}, 227$. doi:10.1016/S0925-4005(01)01009-7

[4] Edmonds, T.E. (1985) Electroanalytical application of carbon fiber electrodes, Analytica Chimica Acta, 175, 1. doi:10.1016/S0003-2670(00)82713-0

[5] Gonon, F., Suaud-Changny, M.F. and Buda, M. (1992) Proceedings of satellite symposium on neu-roscience and technology, Lyon, 215.

[6] Donnet, J.B. and Basal, R.C. (1984) International Fiber Science and Technology, Carbon Fibers, Dekker, New York, 3. 
[7] Decher, G. and Hong, J.D. (1991) Buildup of ultrathin multilayer films by a self-assembly process: II. Consecutive adsorption of anionic and cationic bipolar amphiphiles and polyelectrolytes on charged surfaces. Berichte der Bunsengesellschaft Für Physikalische Chemie, 95, 1430.

[8] Hodak, J., Etchenique, R., Calvo, E.J., Singhal, K. and Bartlett, P.N. (1997) Layer by layer self assembly of glucose oxidase with a poly(allylmanine)-ferrocene redox mediator. Langmuir, 13, 2708.

doi:10.1021/la962014h

[9] Forzani, E.S., Solis, V.M. and Calvo, E.S. (2000) Electrochemical behavior of polyphenol oxidase immobilized in self-assembled structures layer by layer with cationic polyallylamine. Analytical Chemistry, 72, 5300. doi:10.1021/ac0003798

[10] Coche-Guerante, L., Labbe, P. and Mengeand, V. (2001) Analytical Chemistry, 73, 3206. doi:10.1021/ac0015341

[11] Lvov, Y. and Caruso, F. (2001) Biocolloids with ordered urease multilayer shells as enzymatic reactors. Analytical Chemistry, 73, 4212 . doi:10.1021/ac010118d

[12] Fang, M., Grant, P.S., McShane, M.J., Sukhorukov, G. B., Golub, V.O. and Lvov, Y. (2002) Magnetic bio/nanoreactor with multilayer shells of glucose oxidase and inorganic nanoparticles. Langmuir, 18, 6338. doi:10.1021/la025731m

[13] Caruso, F. and Schuler, C. (2000) Enzyme multilayers on colloid particles: Assembly, stability, and enzymatic activity. Langmuir, 16, 9595. doi:10.1021/la000942h

[14] Caruso, F., Fiedler, H. and Haage, K. (2000) Assembly of $\beta$-glucosidase multilayers on spherical colloidal particles and their use as active catalyst. Colloids and Surfaces A: Physicochemical and Engineering Aspects, 169, 287. doi:10.1016/S0927-7757(00)00443-X

[15] Sun, H. and Hu, N. (2004) 1. Voltammetric studies of hemoglobin-coated polystyrene latex bead films on pyrolytic graphite electrodes. Biophysical Chemistry, 110 , 297. sdoi:10.1016/j.bpc.2004.03.005

[16] Rijiravanich, P., Aoki, K. and Chen, J., Surareungchai, W. and Somasundrum, M. (2006) Micro-cylinder biosensors for phenol and catechol based on layer-by-layer immobilization of tyrosinase on latex particles: Theory and experiment. Journal of Electroanalytical Chemistry, 589, 249-258. doi:10.1016/j.jelechem.2006.02.019

[17] Wilcox, D.E., Porras, A.G., Hwang, Y.T., Lerch, K., Winkler, M.E. and Solomon, E.I. (1985) Substrate Analogue Binding to the Coupled Binuclear Copper Active Site in Tyrosinase. Journal of American Chemical Society, 107, 4015. doi:10.1021/ja00299a043

[18] Carbanes, J., Garcia-Canovas, F., Lozano, J.A. and Garcia-Carmona. F (1987) A kinetic study of the melanization pathway between L-tyrosine and dopachrome. Biochimica et Biophysica Acta-General Subjects, 923, 187.

[19] Coely, A., et al. (2001) Backlund and darboux transfor- mation. American Mathematical Society, Providence, RI.

[20] Wadati, M., Sanuki, H. and Konno, K. (1975) Relationships among inverse method, bäcklund transformation and an infinite number of conservation laws. Progress of Theoretical Physics, 53, 419. doi:10.1143/PTP.53.419

[21] Gardener, C.S., Green, J.M., Kruskal, M.D. and Miura, R.M. (1967) Method for solving the Korteweg-de Vries equation. Physical Review Letter, 19, 1095. doi:10.1103/PhysRevLett.19.1095

[22] Hirota, R. (1971) Exact solutions to the equation. describing cylindrical solitons. Physical Review Letter, 27, 1192. doi:10.1103/PhysRevLett.27.1192

[23] Malfliet, W. (1992) Solitary wave solutions of nonlinear wave equations. American Journal of Physics, 60, 650. doi: $10.1119 / 1.17120$

[24] He, J.H. (1998) Approximate analytical solution for seepage flow with fractional derivatives in porous media. Computer Methods in Applied Mechanics and Engineering, 167, 57. doi:10.1016/S0045-7825(98)00108-X

[25] He, J.H. (2005) Approximate solution of nonlinear differential equations with convolution product nonlinearities. Computer Methods in Applied Mechanics and Engineering, 26, 695-700.

[26] He, J.H. (2006) Homotopy perturbation method for solving boundary value problems. Physical Letter A, 350, 87-88. doi:10.1016/j.physleta.2005.10.005

[27] Ariel, P.D. (2010) Homotopy perturbation method and the natural convection flow of a third grade fluid through a circular tube. Nonlinear Science Letters A, 1, 43-52.

[28] Ganji, D.D. and Rafei, M. (2006) Solitary wave solutions for a generalized hirota-satsuma coupled $\mathrm{KdV}$ equation by homotopy perturbation Method. Physical Letter A, 356, 131-137. doi:10.1016/j.physleta.2006.03.039

[29] Meena, A. and Rajendran, L. (2010) Mathematical modeling of amperometric and potentiometric biosensors and system of non-linear equations-Homotopy perturbation approach. Journal of Electroanalytical Chemistry, 644, 50-59. doi:10.1016/j.jelechem.2010.03.027

[30] Anitha, S., Subbiah, A., Rajendran, L. and Ashok K.J. (2010) Solutions of the coupled reaction and diffusion equations within polymer-modified ultramicroelectrodes. Physical Chemistry C, 114, 7030-7037.

[31] Manimozhi, P., Subbiah, A. and Rajendran, L. (2010) Solution of steady-state substrate concentration in the action of biosensor response at mixed enzyme kinetics. Sensor and Actuators B, 147, 290-297. doi:10.1016/j.snb.2010.03.008

[32] Eswari, A. and Rajendran, L. (2010) Analytical solution of steady state current at a microdisk biosensor. Journal of Electroanalytical Chemistry, 641, 35-44. doi:10.1016/i.jelechem.2010.01.015

[33] Eswari, A. and Rajendran, L. (2010) Analytical solution of steady-state current an enzyme-modified microcylinder electrodes. Journal of Electroanalytical Chemistry, 648, 36-46. doi:10.1016/j.jelechem.2010.07.002 


\section{APPENDIX A SYMBOLS USED}

\begin{tabular}{|c|c|c|}
\hline Symbol & Definitions & Units \\
\hline$D_{C}$ & Diffusion coefficient of catechol & $\mathrm{cm}^{2} / \mathrm{s}$ \\
\hline$c_{C}$ & Concentration profile of catechol & mole $/ \mathrm{cm}^{3}$ \\
\hline$c_{E}$ & Concentration profile of enzyme & mole $/ \mathrm{cm}^{3}$ \\
\hline$K_{M}$ & Michaelis Menten constant & $\mathrm{mole} / \mathrm{cm}^{3}$ \\
\hline$K_{\text {cat }}$ & Catalytic rate constant & $\sec ^{-1}$ \\
\hline$c_{Q}$ & Concentration profile of quinone & mole $/ \mathrm{cm}^{3}$ \\
\hline$D_{Q}$ & Diffusion coefficient of quinone & $\mathrm{cm}^{2} / \mathrm{s}$ \\
\hline$c_{C}^{*}$ & Bulk concentration of $C$ & mole $/ \mathrm{cm}^{3}$ \\
\hline$r$ & Radius of the cylinder & $\mathrm{cm}$ \\
\hline$I$ & Current & ampere \\
\hline$r_{0}$ & Electrode radius & $\mathrm{cm}$ \\
\hline$r_{1}$ & Film radius & $\mathrm{cm}$ \\
\hline$r_{1} / r_{0}$ & Dimensionless parameter for film thickness & none \\
\hline$\chi r_{0}$ & Dimensionless parameter for enzyme kinetic & none \\
\hline$j$ & Dimensionless sensor response & none \\
\hline$\psi$ & Dimensionless current & none \\
\hline$C$ & Dimensionless concentration of catechol & none \\
\hline$Q$ & Dimensionless concentration of quinone & none \\
\hline$R$ & Dimensionless distance & none \\
\hline$\gamma_{E}$ & Dimensionless reaction diffusion parameter & none \\
\hline$\gamma_{S}$ & Dimensionless reaction diffusion parameter & none \\
\hline$\alpha$ & Dimensionless saturation parameter & none \\
\hline$L$ & Length of the electrode & $\mathrm{cm}$ \\
\hline$F$ & Faraday constant & $\mathrm{c} \cdot \mathrm{mole}^{-1}$ \\
\hline$n$ & Number of electrons & none \\
\hline
\end{tabular}

\section{APPENDIX B}

Solution of the Eqs.14 and 15 using Homotopy perturbation method. In this appendix, we indicate how Eqs.19 and 20 in this paper are derived. Furthermore, a Homotopy was constructed to determine the solution of Eqs.14 and 15.

$$
\begin{aligned}
& (1-p)\left[\frac{\mathrm{d}^{2} C}{\mathrm{~d} R^{2}}\right]+p\left[\frac{\mathrm{d}^{2} C}{\mathrm{~d} R^{2}}+\frac{1}{R} \frac{d C}{\mathrm{~d} R}-\frac{\gamma_{E} C}{1+\alpha C}\right]=0 \\
& (1-p)\left[\frac{\mathrm{d}^{2} Q}{\mathrm{~d} R^{2}}\right]+p\left[\frac{\mathrm{d}^{2} Q}{\mathrm{~d} R^{2}}+\frac{1}{R} \frac{\mathrm{d} Q}{\mathrm{~d} R}+\frac{\gamma_{S} C}{1+\alpha C}\right]=0
\end{aligned}
$$

and the initial approximations are as follows:

$$
\begin{aligned}
& R=0, C=1, \quad Q=0 \\
& R=\frac{r_{1}}{r_{0}}, C=1, \quad Q=0
\end{aligned}
$$


The approximate solutions of (B1) and (B2) are

$$
C=C_{0}+p C_{1}+p^{2} C_{2}+p^{3} C_{3}+\cdots
$$

and

$$
Q=Q_{0}+p Q_{1}+p^{2} Q_{2}+p^{3} Q_{3}+\cdots
$$

Substituting Eqs.B5 and B6 into Eqs.B1 and B2 and comparing the coefficients of like powers of $p$

$$
p^{1}: \frac{\mathrm{d}^{2} C_{1}}{\mathrm{~d} R^{2}}+\frac{1}{R} \frac{\mathrm{d} C_{0}}{\mathrm{~d} R}-\frac{\gamma_{E} C_{0}}{1+\alpha C_{0}}=0
$$

and

$$
\begin{gathered}
p^{0}: \quad \frac{\mathrm{d}^{2} Q_{0}}{\mathrm{~d} R^{2}}=0 \\
p^{1}: \frac{\mathrm{d}^{2} Q_{1}}{\mathrm{~d} R^{2}}+\frac{1}{R} \frac{\mathrm{d} Q_{0}}{\mathrm{~d} R}+\frac{\gamma_{S} C_{0}}{1+\alpha C_{0}}=0
\end{gathered}
$$

Solving the Eqs.B7 to B10, and using the boundary conditions (B3) and (B4), we can find the following results

$$
\begin{gathered}
C_{0}(R)=1 \\
C_{1}(R)=\frac{\gamma_{E} R^{2}+\gamma_{E}\left(r_{1} / r_{0}\right)-\gamma_{E}\left(1+r_{1} / r_{0}\right) R}{2(1+\alpha)}
\end{gathered}
$$

and

$$
\begin{gathered}
Q_{0}(R)=0 \\
Q_{1}(R)=\frac{\gamma_{S}\left(1+r_{1} / r_{0}\right) R-\gamma_{S}\left(r_{1} / r_{0}\right)-\gamma_{S} R^{2}}{2(1+\alpha)}
\end{gathered}
$$

According to the HPM, we can conclude that

$$
\begin{aligned}
& C(R)=\lim _{p \rightarrow 1} C(R)=C_{0}+C_{1}+C_{2}+\cdots \\
& Q(R)=\lim _{p \rightarrow 1} Q(R)=Q_{0}+Q_{1}+Q_{2}+\cdots
\end{aligned}
$$

Using Eqs.B11 and (B12) in Eq.B15 and Eqs.B13 and B14 in Eq.B16, we obtain the final results as described in Eqs.19 and 20. 